\title{
Integrated Salmonella control in Turkey Production
}

Kilroy $S^{1^{*}}$, Zwaenepoel $P^{2}$, Vangroenweghe $F^{1,3}$

${ }^{1}$ Elanco Benelux, BU Food Animals, Plantijn en Moretuslei $1-3^{\text {rd }}$ floor, 2018 Antwerpen, Belgium

${ }^{2}$ Provet, Talpestraat 2, 8610 Kortemark, Belgium

${ }^{3}$ Ghent University, Faculty of Veterinary Medicine, Dept. Reproduction-Obstretics-Herd Health, Salisburylaan 122, 9820 Merelbeke, Belgium

*Corresponding author: Kilroy S, Elanco Benelux, BU Food Animals, Plantijn en Moretuslei 1 - 3rd floor, 2018 Antwerpen, Belgium

Received date: February 11, 2021; Accepted date: March 11, 2021; published date: March 24,2021

Citation: Kilroy S., Zwaenepoel P., Vangroenweghe F, (2021) Integrated Salmonella control in Turkey Production. Journal of Clinical Research and Reports, 7(2); DOI:10.31579/2690-1919/145

Copyright: () 2021 Kilroy S, This is an open access article distributed under the Creative Commons Attribution License, which permits unrestricted use, distribution, and reproduction in any medium, provided the original work is properly cited.

\begin{abstract}
In recent years, an increase of fattening turkey flocks positive for Salmonella spp. was noted in the European Union. Considering these latest trends, there is a high need to increase knowledge on specific risk factors for fattening turkey flocks in conjunction with successful control measures to combat Salmonella infections on farm. Here, we report a commercial turkey fattening farm that was found positive for Salmonella enterica serovar Typhimurium for two consecutive batches. By means of the Food Safety Program, a risk assessment developed by Elanco (Antwerpen, Belgium) and a farm walk, a Food Safety Index was generated, highlighting specific risk factors. Consequently, an action plan was set up, leading to a favorable increase in the Food Safety Index from $45 \%$ to $67 \%$. Among others, vaccinating fattening turkeys was an important control measure. Monitoring of the Salmonella status of the batches was performed by sampling paper coming from the transport boxes at day of arrival and boot sock samples at the age of 14 weeks. Finally neck skin samples were taken at the slaughterhouse. Uptake of the vaccine was evaluated by sampling of the caeca and taking cloacal swabs 24 hours after first and second vaccination, respectively. Results coming from the analysis of the caeca indicate that vaccination at day one was efficient. Analysis of the cloacal swabs taken after second vaccination was inconclusive. Cooperatively, these actions improved the overall biosecurity of the farm as shown by the negative Salmonella status of the boot sock and neck skin samples in the third batch.
\end{abstract}

Keywords: salmonella; turkeys; biosecurity; vaccines; risk management

\section{Introduction}

Salmonella spp. are one of the major food-borne pathogens and have an importance as a leading cause of food-borne bacterial diseases in humans throughout the world [1]. Different studies indicate that, turkey meat and products are attributed to be important sources of food-borne salmonellosis [2]. The approach against Salmonella spp. in turkey started more than a decade ago. October 2006 marked the start of a European Union (EU)-wide baseline survey over a one-year period, to determine the prevalence of Salmonella spp. in breeding and fattening turkey (Meleagris galapavo) flocks [3]. A decreasing trend in the prevalence of Salmonella-positive flocks was observed in different poultry categories during the period 2007-2018. This decreasing trend however has stabilized over the last 5 years except in breeding turkey flocks, in which the prevalence of Salmonella spp. fluctuated over time [4]. For fattening turkey flocks, in 2019 a total of $6.37 \%$ of flocks were found Salmonella spp. positive in the EU, compared with $5.95 \%$ in 2017 [4]. Based on the results from the latest EU Zoonosis report, it is mandatory to take action in order to decrease Salmonella spp. prevalence in turkeys. Some studies looked at the role processing plays to increase the contamination rate of Salmonella spp. in turkey meat [5,6]. Scalding, de-feathering, evisceration and cooling steps in slaughtering are the critical points in carcass contamination $[5,6]$. Few studies have looked at the risk factors in turkey flocks specifically, which differ from chicken broiler flocks in their seasonality of production and longer growing period. Arsenault et al. reported that turkey flocks were more likely to be infected if they originated from a particular hatchery or were raised in houses where two or more persons had access to [7]. They did not detect association between Salmonella spp. infection at flock level and variables related to pest control, downtime period, manure disposal, and poultry-house cleaning and disinfection practices. Here, we report a commercial fattening turkey farm located in West-Flanders (Belgium) that was found positive for $S$. enterica serovar Typhimurium for two consecutive batches and the methodology used to obtain a negative Salmonella spp. status of the farm. 


\section{Case Description}

\section{Descriptive case information}

Day-of-hatch male and female fattening turkey poults were imported from a French hatchery into Belgium for three consecutive batches. Data regarding the Salmonella status were collected for every batch. The poults were housed on a commercial fattening turkey farm located in WestFlanders (Belgium) comprising 6 houses. Salmonella spp. status of the day-old turkeys was analyzed by collecting paper from the transport boxes as described below. In the third batch, 29000 turkeys were vaccinated on day one (D1) by off-label coarse spray with the commercially available AviPro ${ }^{\circledR}$ Salmonella Duo (Elanco, Cuxhaven-Germany) [8]. Turkey poults were kept in the transport boxes for 15 min after vaccination to increase contact opportunity. A second vaccination was performed at D47 through drinking water. The poults showed no clinical signs nor any adverse events following both vaccination moments.

Sample collection

Samples were collected on a commercial fattening turkey farm with 6 houses located in West-Flanders (Belgium) according to regulation (EC) No 2160/2003; Annex II. For three consecutive batches, 3 papers present in the transport boxes of the day-old poults were analyzed to determine the Salmonella spp. status. Secondary, a pair of boot socks from each house were taken at week 14 to monitor the Salmonella spp. status of the poults. The farm was considered positive when Salmonella spp. were detected in at least one sample. In the third batch, 21 turkeys were randomly selected and euthanized by cervical dislocation 24 hours after spray vaccination to detect the vaccine strains and evaluate the vaccine uptake. The caeca were collected aseptically for vaccine recovery as described below. An additional screening for the Salmonella spp. status of the third batch was performed at the age of 4 weeks by taking 50 cloacal swabs ( 25 males and 25 females). Twenty-four hours after the second vaccination, 25 cloacal swabs (10 males and 15 females) were collected to detect the vaccine strain. Neck skin samples were collected at the slaughterhouse at the age of 16 weeks for the females and 17 weeks for the males.

\section{Bacterial examination of the samples}

Paper present in the transport boxes and boot sock samples were analyzed for Salmonella spp. according to the International Organization for Standardization (ISO 6579-1). Caeca were collected and incubated in Buffered Peptone Water (BPW) for 18 hours ( \pm 2 hours) at $37^{\circ} \mathrm{C}$. Samples were plated directly on Brilliant Green Agar (BGA) plates supplemented with $100 \mu \mathrm{g} / \mathrm{ml}$ rifampicine to detect the vaccine strain. Negative samples were enriched in tetrathionate brilliant green broth by overnight incubation at $37^{\circ} \mathrm{C}$. After incubation, a loopful of the tetrathionate brilliant green broth was plated on BGA supplemented with $100 \mu \mathrm{g} / \mathrm{ml}$ rifampicine. Cloacal swabs taken at week 4 of age were pooled in groups of 5 and analyzed for presence of the Salmonella wild type strains according to the ISO (ISO 6579-1). Cloacal swabs taken 24 hours after second vaccination were pre-enriched in BPW for 18 hours ( \pm 2 hours) at $37^{\circ} \mathrm{C}$ and plated directly on BGA supplemented with $100 \mu \mathrm{g} / \mathrm{ml}$ rifampicine to detect the vaccine strain. Negative samples were enriched in tetrathionate brilliant green broth by overnight incubation at $37^{\circ} \mathrm{C}$. After incubation, a loopful of the tetrathionate brilliant green broth was plated on BGA supplemented with $100 \mu \mathrm{g} / \mathrm{ml}$ rifampicine.

Food Safety Program

A risk assessment focused on live production, Food Safety Program (FSP, Elanco, Antwerpen, Belgium), was used to perform hazard analysis by means of a comprehensive questionnaire and a farm walk. A Food Safety Index (FSI) was created based on the questionnaire, and farm-specific critical control points (CCP) related to the general biosecurity status of the farm were highlighted. The index is presented as a percentage; the higher the percentage, the lower the risk. Following this FSI audit, a report was generated, breaking down all sections and highlighting the CCPs that significantly impacted the risk assessment. The food safety questionnaire and farm visit were performed in January 2020 to establish a baseline FSI. Based on this index, several actions were set up in order to increase the general biosecurity of the farm. The progression and impact of the interventions were evaluated by generating a FSI after control measures were set in place in June 2020. In the frame of the integrated approach, vaccinating fattening turkeys is a critical control measure that is not routinely performed in practice. This study reports the use of a bivalent live attenuated Salmonella vaccine for controlling Salmonella spp. in turkeys.

\section{Results}

Analysis of Salmonella spp. wild type strains

In the period between December 2018 until June 2020, S. enterica serovar Typhimurium was present in the boot sock samples in June 2019 (first batch) and December 2019 (second batch; Table 1).

\begin{tabular}{|l|c|c|c|c|}
\hline \multirow{2}{*}{ Sample } & \multicolumn{2}{|l|}{ Sampling time point } & At slaughter \\
\hline \multirow{2}{*}{ Transport box paper } & $\mathrm{DOA}^{1}$ & 4 weeks & 14 weeks & \\
\cline { 2 - 5 } & $1-, 2-, 3-$ & & $1+, 2+, 3-$ & \\
\hline Boot socks & & & & \\
\hline Cloacal swabs & & $1(\mathrm{NA}), 2(\mathrm{NA}), 3-$ & & $1+, 2+, 3-$ \\
\hline Neck skin & & & & \\
\hline
\end{tabular}

${ }^{1}$ DOA: Day of arrival

Table 1: Results from different sample media at different sampling time points collected for monitoring of Salmonella spp. at the commercial turkey fattening farm. The numbers indicate the results from batch 1, 2 or 3. Negative results are indicated as '-', positive results as ' + '. In case a sampling was not carried out 'NA' (not applicable) is marked. 
Salmonella spp. were also detected through positive neck skin samples collected at slaughter. Consequently, several actions were taken to avoid subsequent Salmonella spp. contamination of the following flock.

\section{Food Safety Index}

Based on the questionnaire and a farm visit, the FSI was set at $45 \%$. The following report highlighted the actions that would lead to a significant increase of the FSI and thus overall biosecurity. The actions are listed in Table 2.

\begin{tabular}{|l|l|}
\hline Biosecurity section & Recommended corrective actions to be applied \\
\hline \multirow{5}{*}{ External farm protection } & repair visible cracks in the houses \\
\cline { 2 - 2 } & increase hand hygiene practices of staff \\
\cline { 2 - 2 } & use dedicated overalls or clothing per individual house \\
\cline { 2 - 2 } & use dedicated footwear and house specific tools \\
\cline { 2 - 2 } & increase rodent control measures \\
\cline { 2 - 2 } & make sure areas around the poultry houses are paved or concreted \\
\hline \multirow{5}{*}{ Internal farm protection } & flush drinking lines \\
\cline { 2 - 2 } & frequent cleaning of the inside of the silo/feed storage \\
\cline { 2 - 2 } & take action to remove beetles present on the farm \\
\cline { 2 - 2 } & use a commercial live vaccine to protect the birds from infection \\
\hline
\end{tabular}

Table 2: Food safety index report highlighting both external and internal biosecurity measures to be improved in order to increase the level of biosecurity for control and prevention of Salmonella spp. at farm level.

After the second batch, litter was removed from the farm immediately after the turkeys were loaded for slaughter. A thorough cleaning with hot water $\left(64^{\circ} \mathrm{C}\right)$ was followed by disinfection with a concentrated disinfectant based on quaternary ammonium, glutaraldehyde, and isopropanol (Virocid ${ }^{\circledR}$; CID Lines, Ieper, Belgium), including the roof top. Additionally, houses were fogged with formaldehyde. The farm premises surrounding the barns were treated with a solution containing $150 \mathrm{~g} / \mathrm{L} \mathrm{NaOCl}$. An aluminum profile was added to the wall at 0,5 meter avoiding migration of beetles to the isolation. Additionally, a concrete wall was constructed at the inside perimeter of the building to prevent external vectors from entering the farm (Figure 1).

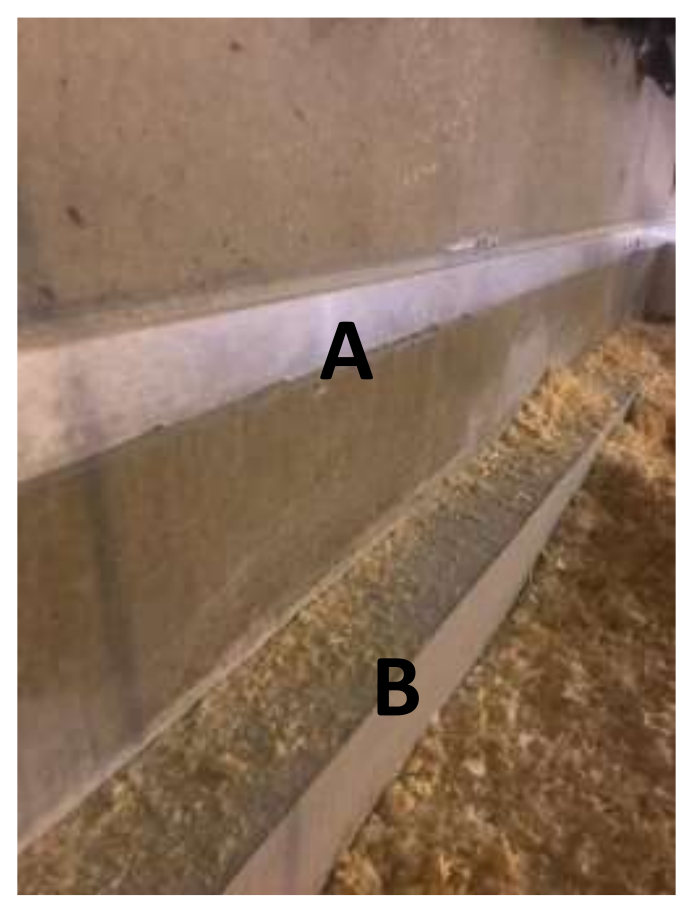

Figure 1:A. Specific control measure to omit beatles from circulation within the building using a sticky glue band; B. Concrete wall constructed at the inside perimeter of the building to prevent external vectors from entering the farm. 
During the third batch, an insecticide containing spinosad (Elektor ${ }^{\circledR}$; Elanco, Antwerpen, Belgium) was applied as described in the directions for use upon arrival of the turkeys. This procedure was repeated at D28. Secondly, the day of hatch turkeys were vaccinated using the commercially available live vaccine AviPro ${ }^{\mathrm{TM}}$ Salmonella Duo (Elanco, Cuxhaven - Germany). The uptake of the vaccine was evaluated by detection of the vaccine strains in the caeca of 21 turkeys, 24 hours after vaccination (Table 3 ).

\begin{tabular}{|l|l|l|}
\hline \multirow{1}{*}{ Sample } & \multicolumn{2}{|l|}{ Sampling time point } \\
\cline { 2 - 3 } & Day 2 & Day 48 \\
\hline Caeca & $21 / 21(100 \%)$ & \\
\hline & & $3 / 25(12 \%)$ \\
Cloacal swabs & & $\delta^{\lambda}: 0 / 10(0 \%) ; \circ: 3 / 15(20 \%)$ \\
\hline
\end{tabular}

Table 3: Analysis results of presence of Salmonella vaccine strains from the commercially applied Salmonella DUO-VAC (Elanco) vaccine in different sample materials and at two different sampling time points to confirm sufficient uptake of the applied vaccine strains. Number of positive samples out of total number of samples collected. If difference exist between male $\left(\delta^{\Uparrow}\right)$ and female $(+)$, sampling results are split in results by sex.

A second vaccination was performed at D47. The uptake of the vaccine was evaluated by collection of cloacal swabs from 25 turkeys. After implementation of the recommended corrective actions another FSP was set up to measure the increase in biosecurity by means of the FSI. The new FSI was set at $67 \%$.

\section{Discussion}

The serotype identified on the commercial farm rearing fattening turkeys in this case report, $S$. enterica serovar Typhimurium, belongs to the top- 6 serovars of fattening turkeys that contribute most to human cases, among $S$. enterica ser. Enteritidis, $S$. enterica ser. Kentucky, S. enterica ser. Newport, S. enterica ser. Virchow and $S$. enterica ser. Saintpaul [2]. Featherstone et al. [9] concluded that, based on a Salmonella spp. testing program, using tap water as a water source and wax blocks to control rodents were effective to reduce the risk of testing positive for $S$. enterica serovar Typhimurium. The results coming from this study indicate that Salmonella spp. contamination originated on farm. The European Food Safety Authority (EFSA) however reported that vertical transmission of Salmonella spp. As well as hatchery acquired Salmonella spp. contamination originating from breeding stock are very important sources for Salmonella spp. infection in turkeys [2]. In order to eliminate Salmonella spp. from a turkey farm, it is necessary to look at the specific risk factors present on farm. By performing a food safety program (FSP), designed by Elanco, these risk factors were highlighted through a comprehensive questionnaire followed by a farm visit. Based on the FSP, a FSI was created before and after a Salmonella spp. control action plan was set up. It was advised to increase external farm biosecurity due to a pig farm located in close proximity of the turkey farm. Indeed, it is widely reported that pigs may act as a source of Salmonella spp. infection [10,11]. Increasing the concrete surface of the surroundings and cleaning with a solution containing $150 \mathrm{ng} / \mathrm{L} \mathrm{NaOCl}$ on a timely basis could prevent manifestation of Salmonella spp. on the farm premises. Using farm specific materials, such as clothing and boots, together with improved hand hygiene practices for staff members also improved external as well as internal farm biosecurity. A concrete wall was built to avoid infestation of rodents and other possible vectors entering the houses. The role of mice and other rodents in Salmonella spp. epidemiology on poultry farms is well documented [12, 13].

During the farm visit beetles were detected in the houses. An insecticide containing spinosad (Elektor ${ }^{\circledR}$; Elanco) as the active ingredient was implemented upon arrival of the day-old turkeys. The treatment was repeated at week 4 . Since Salmonella spp. among other pathogens might reside within biofilms, flushing the drinking lines improved water quality. Cleaning silo bins on a timely basis corroborates ingestion of Salmonella spp. free feed. Since no other farms were reported Salmonella positive supplied by the same feed mill, it was assumed the feed was Salmonella free. Featherstone et al. previously identified additional risk factors for Salmonella spp. infection on fattening turkey units, based on a crosssectional study [10]. Seasonal flock production had an odds of 0.33 to contract Salmonella spp. as compared to flocks produced in year-round consecutive batches. Indeed, an extended period between batches of birds is optimal for thorough cleaning and disinfection and interrupts the carryover of infection between consecutive batches. Other studies have identified the hatchery as risk factor for Salmonella spp. in poultry [14] Systematically, these actions improved the overall biosecurity of the farm. Importantly, the number of beetles was reduced, indicating that the insecticide was efficient at killing the residing beetle population. When biosecurity is at an acceptable level, vaccination with a commercially available live vaccine strain can protect the birds against Salmonella spp. infection. Incontrovertibly, the level of protection generated by vaccination not only depends on the challenge itself (e.g. strain involved, infection dose, route of transmission), but is also influenced by the route of vaccine administration, the vaccine dose, vaccine preparation, water quality, age of the birds and genetics [15]. Therefore, scientific evidence indicates that vaccination will be more efficient in protecting a flock when environmental presence of Salmonella spp. is duly managed by complementary measures reducing the infection pressure over time. Moreover, live Salmonella vaccines generally confer better protection than killed vaccines, because the former stimulate both cell-mediated and humoral immunity [16]. Since infection with Salmonella spp. usually occurs in the first week of life, it is important to vaccinate as soon as possible. This is supported by additional data indicating that infection of very young chicks results in high levels of environmental contamination [17]. In this study, day-old turkeys were vaccinated upon arrival, followed by drinking water vaccination at D47 of age. Opening the vaccine under water and stirring for a few minutes prior to spraying the water solution containing the vaccine improves the homogenic spread of the vaccine in the spray solution. Allowing the day-old turkeys to stay in close contact in the transport boxes for 15 minutes increased ingestion and guaranteed a successful vaccination, as shown by the positive caeca samples of 21 
randomly selected turkeys. Although spray vaccination is currently off label, it has been previously demonstrated that spray vaccination of day old chicks is a practical way to ensure ingestion of the vaccine at an early age [8]. A second dose of the vaccine was administered at D47, conferring resistance not only in the immediate post-hatch period but also maintaining longer-term protective effects. Due to the nature of both vaccine strains having a decreased shedding over time, it is less recommended to take cloacal swabs to monitor the uptake of the vaccine strain by the turkeys, as shown under experimental conditions with broilers [8]. Combining increased biosecurity together with bivalent live vaccination, enabling active immunity against target serovars, was successful in protecting turkeys from Salmonella spp. infection. Vaccination is likely to become increasingly important in the control of Salmonella spp. for the foreseeable future, especially in turkeys. Since most Salmonella spp. infections are subclinical, it is recommended to continue monitoring for Salmonella spp. presence on the farm, allowing prompt detection and response to Salmonella spp. infections. A testing program will also help to further develop action plans to avoid spread and persistence of Salmonella spp. infections.

\section{Conclusions}

In conclusion, this study describes important practical measures that can be implemented by farmers and veterinarians within the turkey industry to control salmonellosis, although additional risk factors at other turkey farms should be taken into account. Finally, Salmonella spp. bivalent live vaccination should be considered as a critical part of the package of control measures to limit $S$. enterica serovar Typhimurium infection in turkeys when biosecurity is at an acceptable level.

\section{ACKNOWLEDGEMENTS}

Conceptualization, S.K and P.Z; Methodology, S.K and P.Z; WritingOriginal Draft Preparation, S.K, P.Z and F.V.; Writing-Review \& Editing, F.V.

\section{References}

1. D’Aoust J, Maurer J. Salmonella species. Food Microbiol: Fundamentals and Frontiers 2007; 3rd edition: p. 187-236.

2. European Food Safety Authority and European Centre for Disease Prevention and Control (EFSA and ECDC). The European Union summary report on trends and sources of zoonoses, zoonotic agents and food-borne outbreaks in 2010. EFSA J. 2012; 10(2597), p.442.

3. Anonymous. Report of the task force on zoonoses data collection on the analysis of the baseline survey on the prevalence of Salmonella in turkey flocks. Part A: Salmonella prevalence estimates. EFSA J. 2008; 134, 1-91.

4. European Food Safety Authority and European Centre for Disease Prevention and Control (EFSA and ECDC). The European Union One Health 2018 Zoonoses Report. EFSA J. 2019; 110, 2-76.
5. Nde CW, Sherwood JS, Doetkott C, Logue CM. Prevalence and molecular profiles of Salmonella collected at a commercial turkey processing plant. J Food Prot. 2006; 69(8), 1794-1801.

6. Allen VM, Tinker DB, Hinton MH, Wathes CM. Dispersal of microorganisms in commercial defeathering systems. Br Poult Sci. 2003; 44, 53-59.

7. Arsenault J, Letellier A, Quessy S, Morin JP, Boulianne M. Prevalence and risk factors for Salmonella and Campylobacter spp. carcass contamination in turkeys slaughtered in Quebec, Canada. J Food Prot. 2007; 70(6), 1350-1359.

8. De Cort W, Haesebrouck F, Ducatelle R, van Immerseel F. Administration of a Salmonella Enteritidis $\Delta$ hilAssrAfliG strain by coarse spray to newly hatched broilers reduces colonization and shedding of a Salmonella Enteritidis challenge strain. Poult Sci. 2015; 94(1), 131-135.

9. Featherstone CA, Reichel R, Snow LC, Davies RH. Investigation of risk factors for Salmonella on fattening-turkey farms. Epidemiol Infect. 2010; 138(10), 1427-1438.

10. Campos J, Mourão J, Peixe L, Antunes P. Nontyphoidal Salmonella in the Pig Production Chain: A Comprehensive Analysis of Its Impact on Human Health. Pathogens. 2019; 8(1), 19.

11. Bonardi S. Salmonella in the pork production chain and its impact on human health in the European Union. Epidemiol Infect. 2017; 145, 1513-1526.

12. Henzler DJ, Opitz HM. The role of mice in the epizootiology of Salmonella enteritidis infection on chicken layer farms. Avian Dis. 1992; 36(3), 625-631.

13. Greig J, Rajić A, Young I, Mascarenhas M, Waddell L, LeJeune J. A scoping review of the role of wildlife in the transmission of bacterial pathogens and antimicrobial resistance to the food Chain. Zoonoses Public Health. 2015; 62(4), 269-284.

14. Papadopoulou C, Carrique-Mas JJ, Davies RH. Salmonella in turkey flocks in Great Britain - a review. Proceedings of 7 th International Symposium on Turkey Diseases, Berlin, Germany, 2008; 19-21, pp. 264-273.

15. Kilroy S, Raspoet R, Devloo R, Haesebrouck F, Ducatelle R, Van Immerseel F. Oral administration of the Salmonella Typhimurium vaccine strain Nal2/Rif9/Rtt to laying hens at day of hatch reduces shedding and caecal colonization of Salmonella 4,12:i:-, the monophasic variant of Salmonella Typhimurium. Poult Sci. 2015; 94(6), 1122-1127.

16. Babu U, Scott M, Myers MJ, Okamura M, Gaines D, Yancy HF, Lillehoj H, Heckert RA, Raybourne RB. Effects of live attenuated and killed Salmonella vaccine on T-lymphocyte mediated immunity in laying hens. Vet Immunol Immunopathol. 2003; 91(1), 39-44.

17. Pande VV, Devon RL, Sharma P, McWhorter AR Chousalkar KK. Study of Salmonella Typhimurium Infection in Laying Hens. Frontiers in Microbiology. 2016; 7: 203. 
This work is licensed under Creative Commons Attribution 4.0 License

To Submit Your Article Click Here: Submit Article

DOI:10.31579/2690-1919/145
Ready to submit your research? Choose Auctores and benefit from:

* fast, convenient online submission

* rigorous peer review by experienced research in your field

* rapid publication on acceptance

* authors retain copyrights

* unique DOI for all articles

* immediate, unrestricted online access

At Auctores, research is always in progress.

Learn more www.auctoresonline.org/journals/journal-of-clinicalresearch-and-reports 